\title{
Re-anatomization of Conoid and Deciduous Lateral Incisors with Direct Composite Resin
}

\author{
${ }^{1}$ Carolina Santezi, ${ }^{2}$ Janaína Freitas Bortolatto, ${ }^{3}$ Michael Christopher Floros \\ ${ }^{4}$ Marcelo Ferrarezi de Andrade, ${ }^{5}$ Lívia Nordi Dovigo
}

\begin{abstract}
Discrepancies in tooth size and shape are commonly encountered in the dental clinic, and the conoids lateral incisors are among the most affected. This anomaly affects approximately $1 \%$ of the population and it strongly influences the disharmonic characteristics in the smile. This paper reports a clinical case describing a correct diagnosis, planning and the subsequent esthetic treatment of asymmetric lateral incisors using direct composite resin.
\end{abstract}

Keywords: Composite resin, Conoid teeth, Deciduous teeth, Re-anatomization.

How to cite this article: Santezi C, Bortolatto JF, Floros MC, de Andrade MF, Dovigo LN. Re-anatomization of Conoid and Deciduous Lateral Incisors with Direct Composite Resin. World J Dent 2016;7(1):41-46.

\section{Source of support: Nil}

Conflict of interest: None

\section{INTRODUCTION}

Tooth agenesis is one of the most prevalent anomalies in the dental clinic ${ }^{1}$ and is characterized by congenital absence of one or more teeth. Much of the researches on agenesis suggest that women are more affected than men, and the most common etiological factor is heredity. ${ }^{1-7}$ The permanent dentition is usually affected by this numerical variation, and the most affected teeth are the upper lateral incisors, followed by premolars and third molars. ${ }^{8,9}$ Around $2 \%$ of the patients who look for

${ }^{1} \mathrm{PhD}$ Student, ${ }^{2,3}$ Postdoctoral Fellow, ${ }^{4}$ Adjunct Professor

${ }^{5}$ Assistant Professor

${ }^{1,5}$ Department of Social Dentistry, Araraquara Dental School Universidade Estadual Paulista, Araraquara, São Paulo, Brazil

${ }^{2}$ Department of Biomaterials, University of Toronto, Toronto Canada

${ }^{3}$ Department of Trent Centre for Biomaterials Research, Trent University, Peterborough, Canada

${ }^{4}$ Department of Restorative Dentistry, Araraquara Dental School Universidade Estadual Paulista, Araraquara, São Paulo, Brazil

Corresponding Author: Carolina Santezi, PhD Student, Department of Social Dentistry, Araraquara Dental School Universidade Estadual Paulista, Humaitá Street, 1680, 14801-903 Araraquara São Paulo, Brazil, Phone: 551633016552, e-mail: carolina santezi@hotmail.com treatment of congenital dental anomalies seek correction of permanent upper lateral incisors, ${ }^{1,7,10,11}$ which may be uni- or bilaterally absent. When agenesis of permanent teeth occurs unilaterally in the arch, it is often associated with a shape and size discrepancy on the contralateral upper lateral incisor. ${ }^{1,11}$ Indeed, the literature shows that patients with tooth agenesis seem to have others teeth malformations, ${ }^{1-11}$ such as the existence of conoid lateral incisor. ${ }^{1,3,6}$ Conoid teeth are characterized by a cone-shaped coronary anatomy, with the apex located in the incisal. Some authors found a higher frequency of this type of anomaly in women, ${ }^{12,13}$ with the highest prevalence occurring in the permanent dentition ${ }^{14}$ in the upper left region. ${ }^{13,15}$ Due to the conicity, such anomaly is one of the primary reasons for the presence of maxillary anterior diastema, ${ }^{16-20}$ as it produces a significant reduction of the tooth structure, especially in the mesiodistal surfaces. Agenesis and anomalies of shape located in the anterior region of the upper dental arch generally lead to unappealing esthetics and possible phonetic problems. ${ }^{21,22}$ Patients with agenesis and abnormally shaped upper lateral incisors often seek esthetic treatments due to the large disharmony in their smile, since the size of the teeth and the height-width proportion of the crown are among the most evident factors contributing to desirable esthetics. ${ }^{16,17}$ These factors directly influence the social life of the individual, ${ }^{7}$ and the main purpose of these esthetic and cosmetic dental procedures is to obtain the best esthetic results so the patient can have a more desirable smile, ${ }^{23}$ improving confidence and the ability to integrate socially.

A simple and effective solution for the treatment of tooth shape and size disharmony is the restorative treatment with direct composite resin, 19,20,23-28 which retains the dental structure, provides a short treatment time with a lower cost to the patient, is reversible and the material can be resized or reshaped at a future time if necessary. The technique recommended for this procedure employs an intermediate stage which gives the patient a previous idea of the final esthetic and functional result. This allows the patient to actively evaluate the ongoing results for their rehabilitation, and they may critically evaluate the functionality and appearance, with the ability to propose changes for the final step, 
improving their satisfaction. For this intermediate step, a transparent silicone tray filled with bis-acryl resin is generally constructed, which is light cured through the tray, resulting in a temporary restoration quite similar to the final, permanent restoration (MockUp). ${ }^{28}$ However, this procedure adds significant cost, making it impractical for many situations. Considering the importance of this step for the success of esthetic treatment, the aim of this paper is to describe a clinical case report for the treatment of dental agenesis with anomaly of shape in the contralateral upper lateral incisor, highlighting the use of an alternative and more accessible technique for obtaining high quality and inexpensive Mock-Ups.

\section{CASE REPORT}

A 27-year-old female patient presented to the restorative dentistry clinic at Araraquara School of Dentistry (Universidade Estadual Paulista) with complaints of dissatisfaction with the shape and appearance of their conoid upper left lateral incisor and deciduous upper right lateral incisor. During the anamnesis, clinical and radiographic examinations, possible periapical and periodontal alterations were ruled out. Nevertheless, the hypodontia of the left upper lateral incisor (conoid) and the agenesis of the right upper lateral incisor, with the corresponding deciduous remaining with good alveolar implantation, were observed (Figs $1 \mathrm{~A}$ to C). Additionally, class II, division 2 malocclusion was diagnosed according to Angle's classification. No articular disorder was present and the patient had no pain or difficulty when performing openings and closings as well as protrusion and lateral movements. After diagnosis, a multidisciplinary treatment plan between the departments of restorative dentistry and orthodontics was formulated, but the patient declined the recommended orthodontic treatment, so a modified treatment strategy was required. A restorative cosmetic treatment was selected involving a selective vestibular grinding for 'repositioning' of proclined teeth [left and right upper lateral incisors (Figs 2A and B)] in the arch and reshaping of them with direct composite resin. Two in-office tooth bleaching sessions, with a week interval between them, were performed prior to the restorative treatment. After the two bleaching sessions, the final color of teeth was A1 (Figs 3A to C). A condensation silicone impression of the upper and lower arches was performed in order to obtain dental casts and to do the diagnostic wax-up. Silicone trays similar to those used for at-home tooth bleaching were used to realize the restorative test [Mock-Up (Figs 4A and B)]. Then the vestibular surfaces of the right and left upper lateral incisors were carefully worn out using a diamond bur 4138 (KG Sorensen) according to the inclination of the three coronary thirds and controling the wear by means of a condensation silicone guide as shown before in Figure 2. After completing the grinding, a flowable composite resin (Natural Flow-DFL; A1 color) was inserted into the previously prepared silicon impression tray. The impression tray was positioned on the upper arch for light curing (the resin in the regions of the right and left upper lateral incisors (40 seconds per face using Bluephase C8-Ivoclar vivadent with light intensity of $800 \mathrm{~mW} / \mathrm{cm}^{2} \pm 10 \%$ ). Cervical excesses of provisional restorative material were removed with a number 12 scalpel, and a polishing with finishing disks (Soflex 3M ESPE) was conducted (Figs $5 \mathrm{~A}$ to D). The patient was
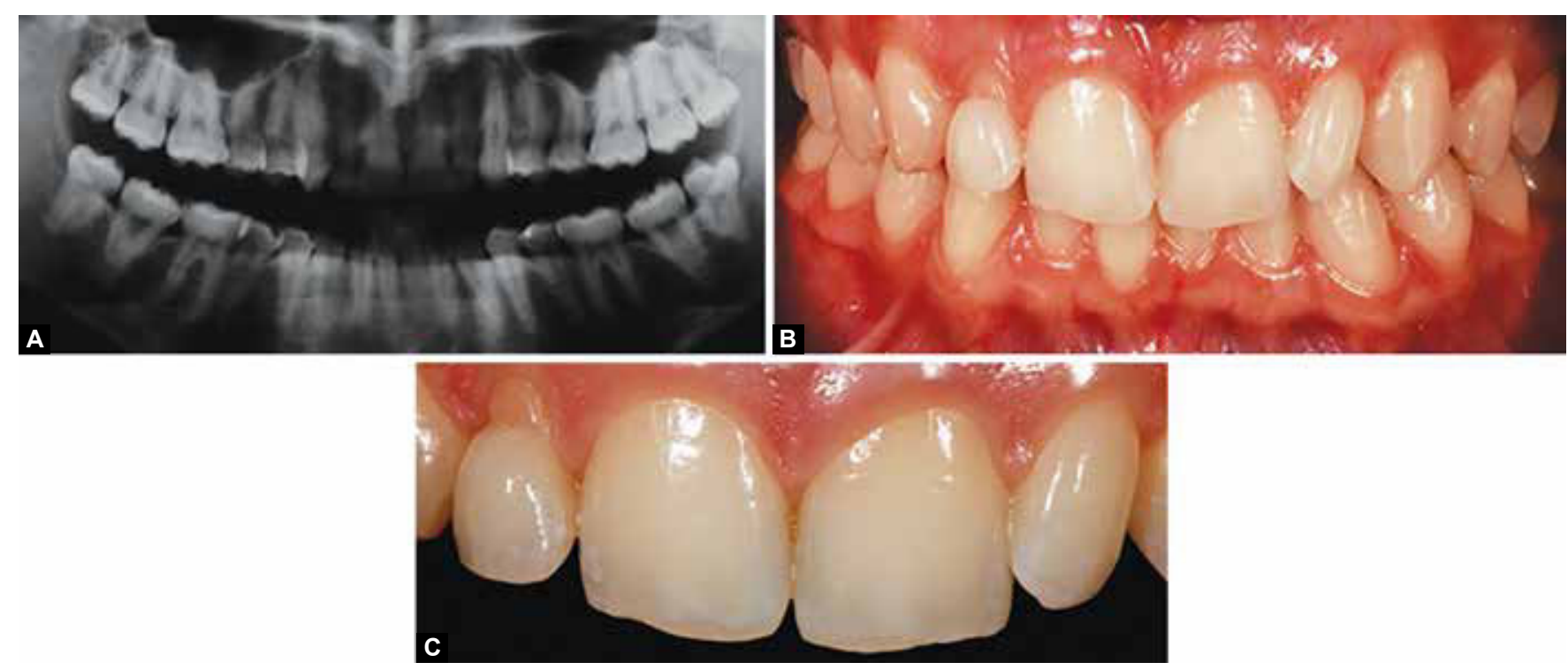

Figs $1 \mathrm{~A}$ to $\mathrm{C}$ : (A and $\mathrm{B})$ Respectively radiographic and clinical examinations, it is possible to see hypodontia of the left upper lateral incisor and agenesis of the right upper lateral incisor with the corresponding deciduous remaining with good alveolar implantation and (C) a closer view of the dental anomalies 

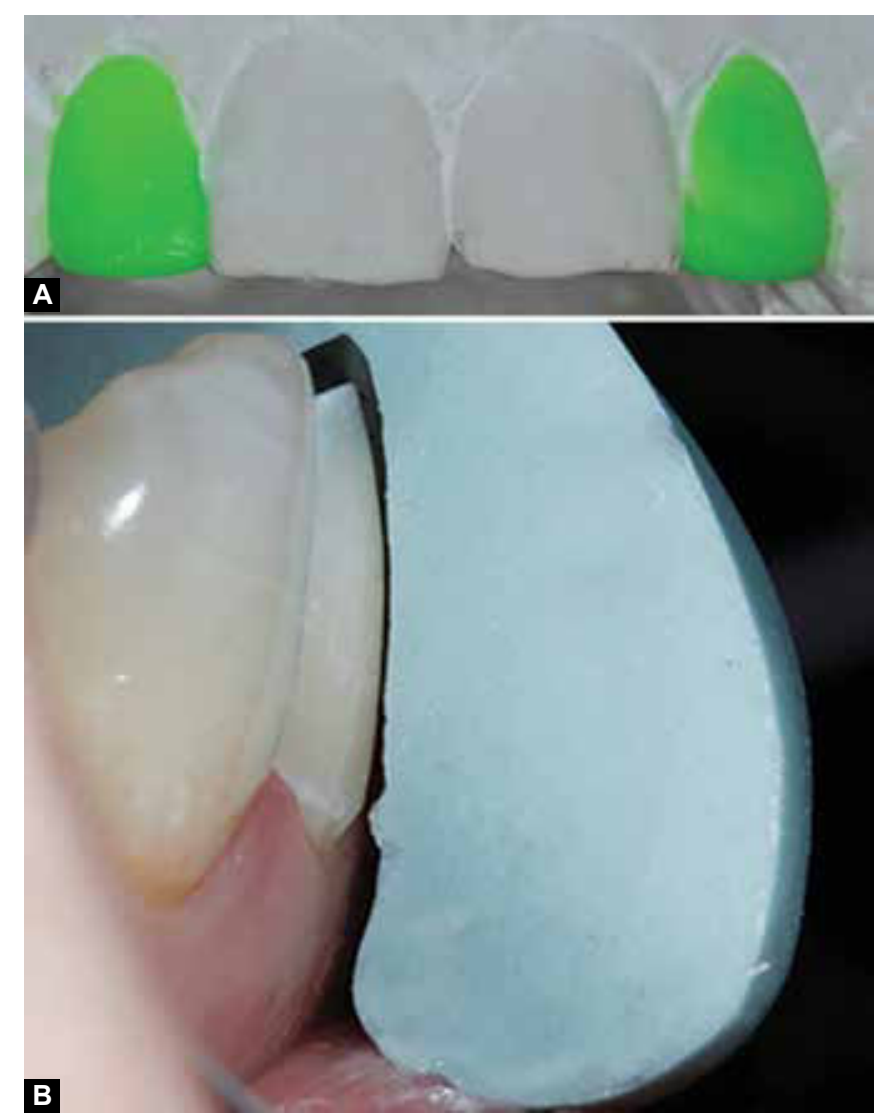

Figs 2A and B: (A) Diagnostic wax-up of the 52 and 22 elements and $(B)$ the silicone matrix positioned in the upper arch to perform the selective grinding of teeth

advised to critically evaluate her temporary smile to confirm the desired appearance and functionality for the final restoration. She also received guidance on the care necessary to maintain the temporary restoration until the next appointment.

In the subsequent appointment, the temporary restoration was removed with the aid of a hollemback (Fig. $6 \mathrm{~A})$. It was followed by dental prophylaxis with pumice powder and water. Immediately after, a relative isolation was fitted (Fig. 6A). Adjacent teeth were protected using Teflon tape (Fig. 6B). A 37\% phosphoric acid gel was used to etch the surfaces of right and left upper lateral incisors for 15 and 30 seconds in enamel and dentin, respectively (Ultra etching $37 \%$-Ultradent) (Fig. 6B). After washing with water to remove the phosphoric acid and the tape removal, a new relative isolation was placed. Then, the retraction cord \#000 (Ultrapack 000-Ultradent) was inserted into the gingiva of the teeth to be restored and a new tape was positioned surrounding the collateral teeth (Fig. 6D). The same was done on the contralateral side (Fig. 6D). Primer and adhesive were applied (Adper Scotchbond Multi-Purpose-3M ESPE) with subsequent light curing (20 seconds per face). Thereafter, with the aid of an intraoral silicone guide, the palatal enamel was built using a composite resin shade color A1 Enamel (Z350 Filtek Supreme XT-3M ESPE) (Fig. 7A). For this, a thin layer of material was placed on the guide and it was taken in position with subsequent light curing (40 seconds). To build the artificial dentin, a thin opaque composite resin layer (A1 Dentin Z350 Filtek Supreme XT-3M ESPE) was inserted and, prior to curing, a composite resin for incisal effect (UV Z350 Filtek Supreme XT—3M ESPE) was placed
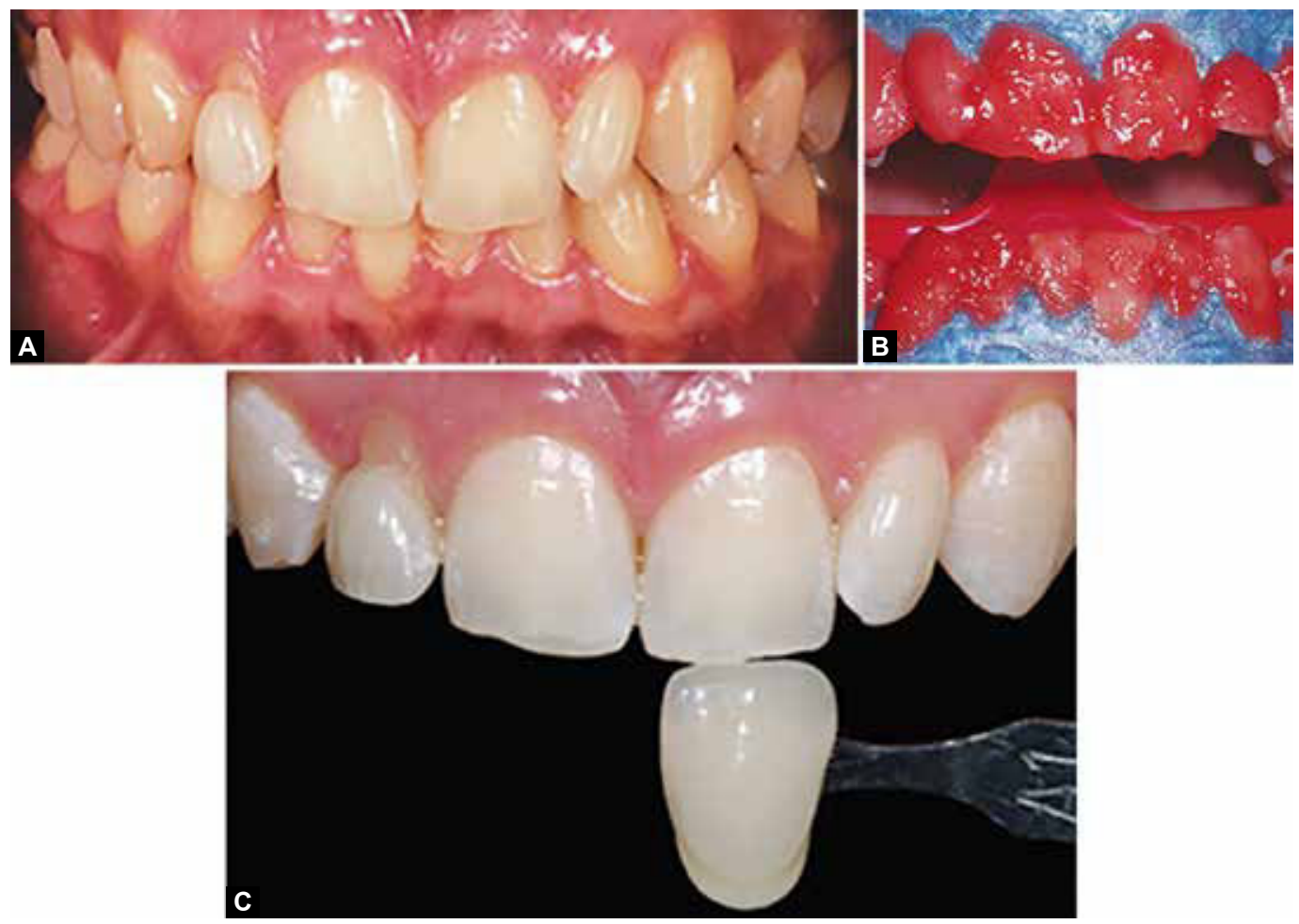

Figs $3 A$ to $C:(A)$ Initial color of teeth, $(B)$ during dental bleaching in office and (C) final color of teeth (A1) 


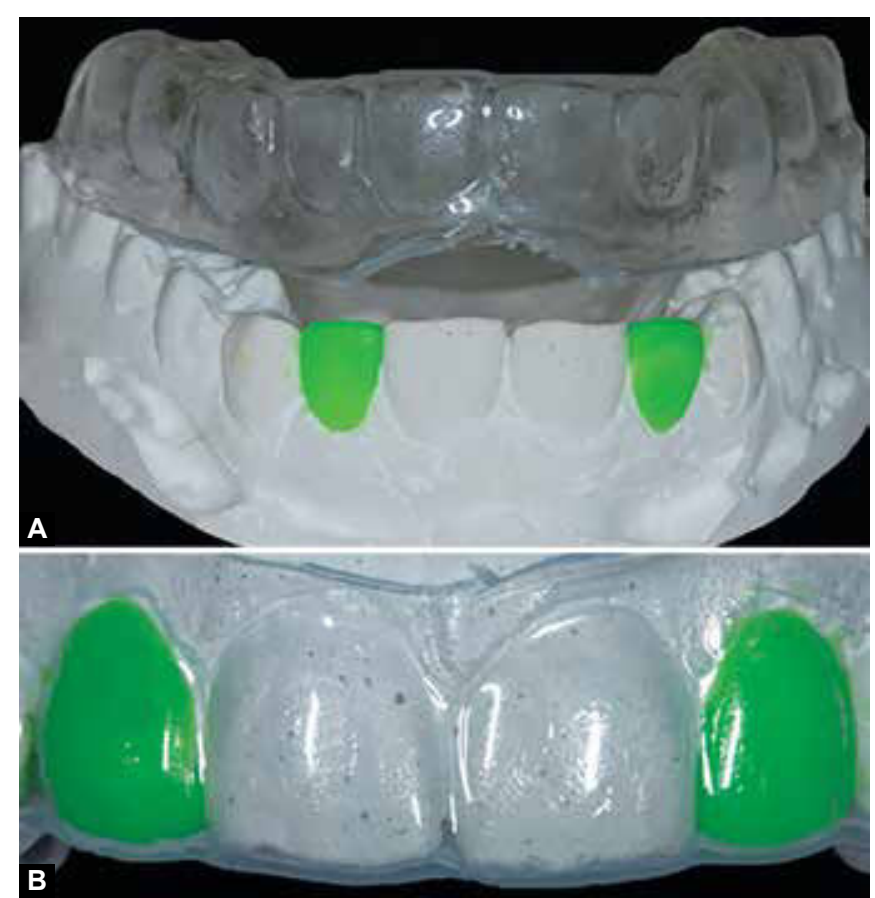

Figs 4A and B: Silicone impression tray obtained from the diagnostic wax-up: $(A)$ the silicone impression tray is out of the model and $(B)$ positioned in the plaster model showing the adaptation

on the proximal and incisal edges of the teeth (Fig. 7A). Subsequently, a composite resin color A1 Enamel (Z350 Filtek SupremeXT-3M ESPE) was used to build the labial enamel surface and the final light curing was carried out (40 seconds) (Fig. 7B). The retraction cord was removed and the labial and lingual faces were finished with Diamond Burs 2135FF and 3118FF (KG Sorensen) respectively.
A week later, the final polishing was performed with Soflex Pop-on (3M ESPE) finishing and polishing disks, followed by a diamond paste impregnated felt disk (Enamelize-Cosmedent) and finally a silicon carbide brush was used. The final result is shown in Figure 8 and the result after 2 years follow-up can be seen in Figure 9.

\section{RESULTS}

The obtained result was satisfactory to the professionals and very satisfactory to the patient (Fig. 8). As the grinding was large and the re-anatomization was made by direct technique, the authors followed-up the case for 2 years and the result can be seen in Figure 9.

\section{DISCUSSION}

It is increasingly common for patients to request cosmetic procedures, as an esthetically pleasing smile has a direct impact on an individual's social life. For this reason, it is extremely important that dental professionals are able to restore not only functionality, but also desirable esthetics. ${ }^{29}$ The main treatment options for diastema closure derived from incisors with agenesis and/or shape anomaly are re-anatomization by direct restoration using composite resin and indirect ceramic or ceromer veneers.

The development of adhesive materials has enabled the more conservative dentistry and improved esthetic results. Recent advances in dental materials allow reproduction of dentin, enamel and individual tooth characteristics to an almost imperceptible degree. Current
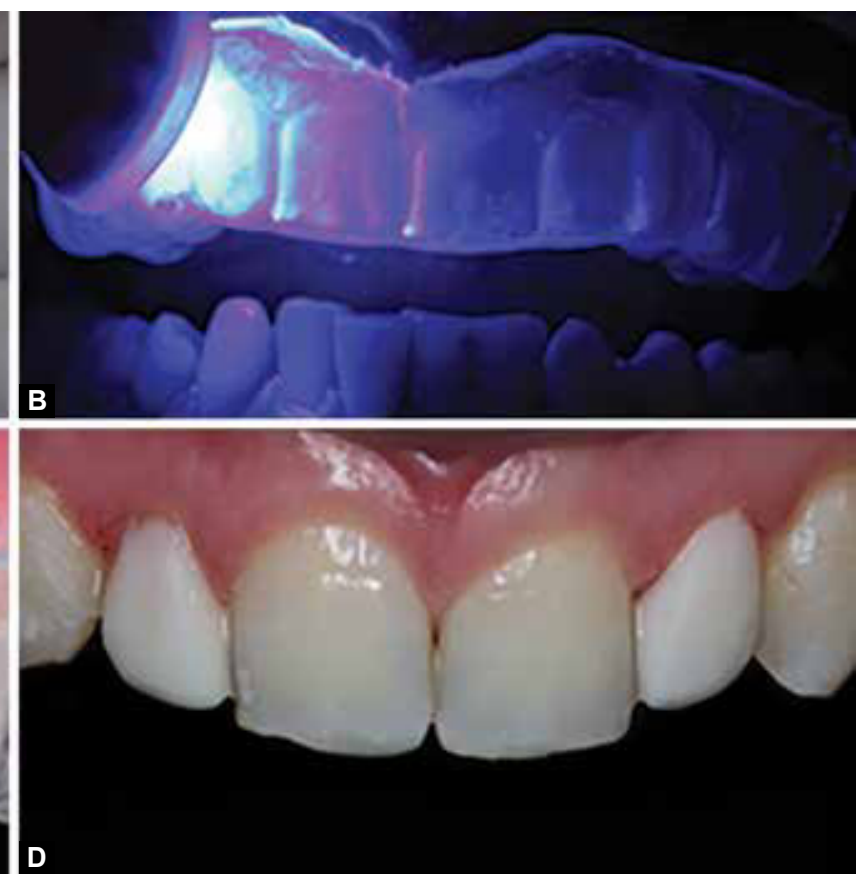

Figs 5A to D: The impression tray was filled with a flowable composite resin (Natural flow-DFL; A1 color) as it is possible: (A and B) the impression tray was positioned on the upper arch for light curing (40 seconds per face using), (C) the result immediately after light curing and (D) results from the removal of the cervical excesses with a number 12 scalpel, and polishing with finishing disks (Soflex 3M ESPE) 


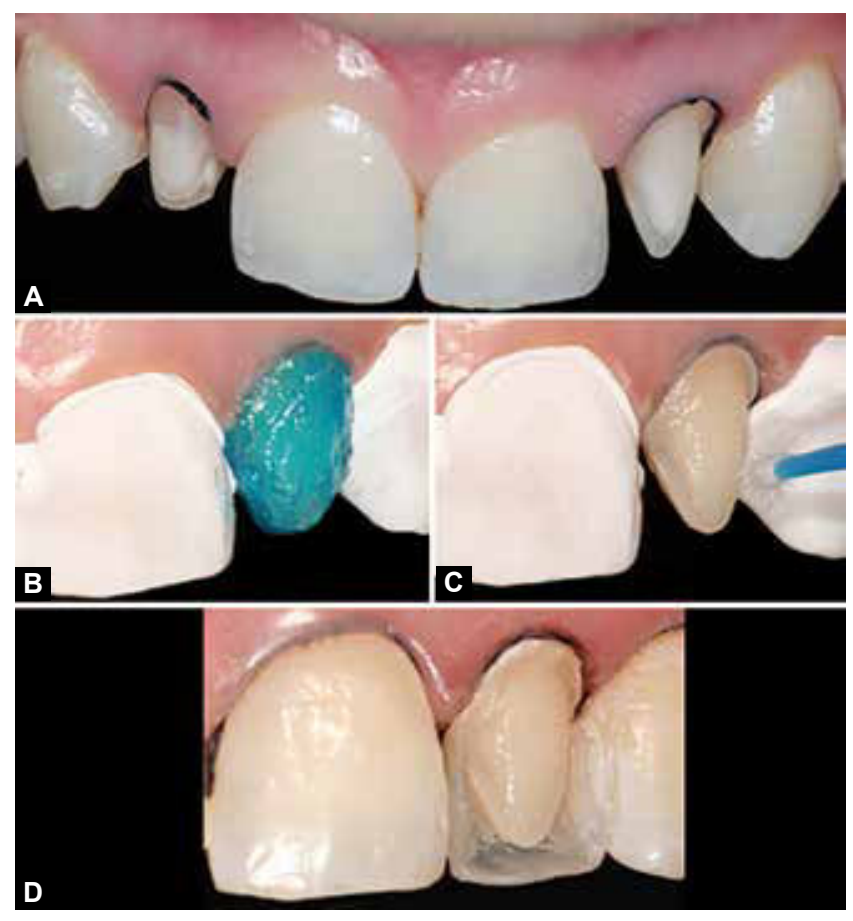

Figs 6 A to D: (A) The grinding of teeth. After that, the protection tape on collateral teeth were positioned and the teeth surfaces were conditioned for 15 and 30 seconds in enamel and dentin respectively, (B) (37\% phosphoric acid gel; ultra etching 37\%Ultradent), (C) the placement of the retraction cord (Ultrapack 000-Ultradent) and application of the primer and bond (Adper Scotchbond Multipurpose-3M ESPE); after light curing ( 20 seconds per face), the construction of the palatal enamel (A1 Enamel; Z350 Filtek Supreme XT-3M ESPE) was made as it is possible to see and $(D)$ the same procedures were realized in the contralateral tooth

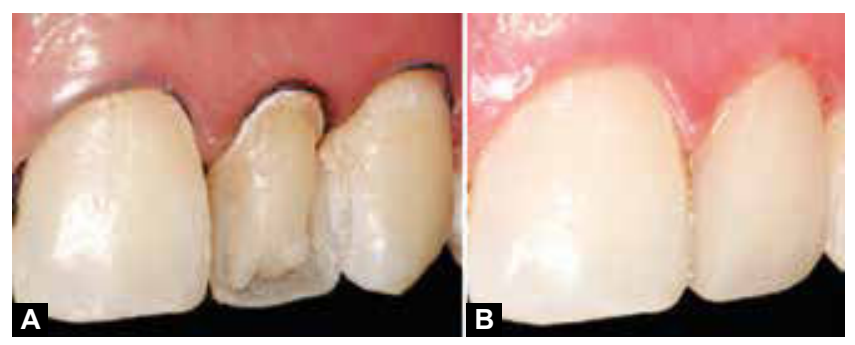

Figs 7A and $B$ : (A) The artificial dentin been mimetized using an opaque composite resin layer (A1 Dentin Z350 Filtek Supreme XT3M ESPE), followed by insertion of composite resin for incisal effect (UV Z350 Filtek Supreme XT-3M ESPE) on the proximal and incisal edges of the teeth and the construction of the labial enamel surface (A1 Enamel; Z350 Filtek Supreme XT-3M ESPE). After that, light curing for 40 seconds was performed and the excess was removed and (B) result immediately after tooth reconstruction, the same procedures were realized in the contralateral tooth

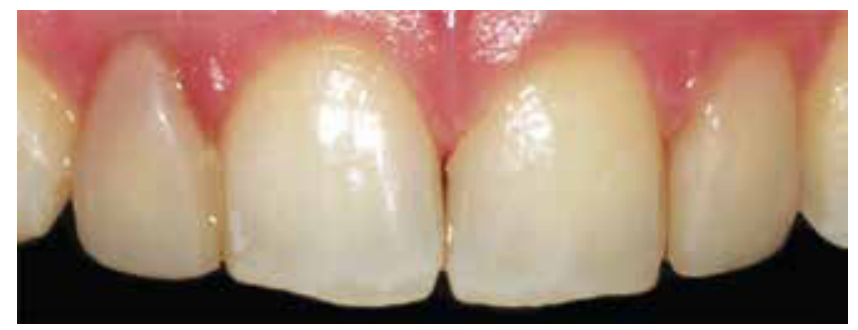

Fig. 8: Result immediately after finishing and polishing using Soflex Pop-on (3M ESPE) disks, followed by a diamond paste impregnated felt disk (Enamelize-Cosmedent) and a silicon carbide brush

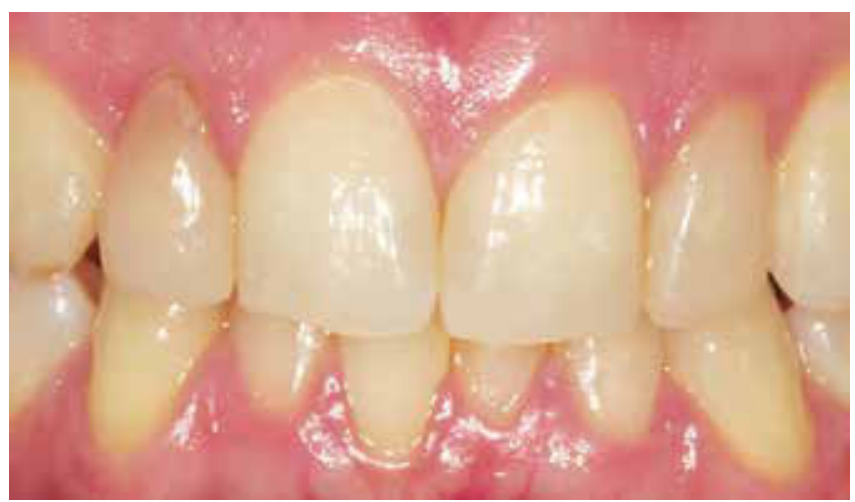

Fig. 9: Result after 2 years follow-up

composite resins are available in many different colors, with differing intensities and degrees of luminosity, allowing mimic of optical characteristics of natural teeth as fluorescence, opalescence and translucency, which is extremely important when it comes to restorations in anterior teeth. ${ }^{18,29-32}$

The best treatment plan is the one that takes into consideration the different socioeconomic realities of patients in association with dental materials and techniques available. Most of the materials used in cosmetic dentistry have high costs which are invariably transferred to the patient. In this work, we proposed a way to minimize the cost replacing the transparent silicone tray and bisacryl resin by an inexpensive silicon tray made from the diagnostic wax-up and standard flowable composite resin.

Because the patient did not have financial conditions to afford private care and was being attended to in the public facilities of the Araraquara School of DentistryUNESP, this alternative was able to remedy the esthetic concerns of the patient without sacrificing the final quality of work. It is worth mentioning that the final result was extremely satisfying for the patient and that 3 years after the procedure, the restorations remain esthetically pleasing. It is therefore important to emphasize that the esthetic excellence can be achieved with alternative quality treatments. Dentists are responsible for technical improvement and creativity necessary to ensure patient satisfaction. We have herein described a procedure using commonly available and inexpensive materials to re-anatomization a smile without reducing treatment quality and, improving the individual in the broader context of 'health', where not only physical health is returned, but also mental and social aspects. ${ }^{33}$

\section{REFERENCES}

1. Woodwhorth DA, Sinclair PM, Alexander RG. Bilateral congenital absence of maxillary lateral incisors: a craniofacial and dental cast analysis. Am J Orthod 1985 Apr;87(4):280-293. 
2. McNeill RW, Joondeph DR. Congenitally absent maxillary lateral incisors: treatment planning consideration. Angle Orthod 1973 Jan;43(1):24-29.

3. Hoo JJ. Anodontia of permanent teeth and pegged missing maxillary lateral incisors in the same family. Am J Med Genet $2000 \mathrm{Feb} ; 90(4): 326-327$.

4. Ahmad W, et al. A locus for autosomal recessive hypodontia with associated dental anomalies maps to chromosome 16q12.1. Am J Hum Genet 1998 Apr;62(4):987-991.

5. Atasu A, Akyus S. Congenital hypodontia: a pedigree and dermatoglyphic study. J Clin Pediatr Dent 1995 Spring MarJun;19(3):215-224.

6. Dermaut LR, Goeffers KR, De Smit AA. Prevalence of tooth agenesis correlated with jaw relationship and dental crowding. Am J Orthod Dentofac Orthop 1986 Sep;90(3): 204-210.

7. Robertsson S, Mohli NB. The congenitally missing upper lateral incisor: a retrospective study of orthodontic space closure versus restorative treatment. Eur J Orthod 2000 Dec; 22(6):697-710.

8. Graber LW. Congenital absence of teeth: a review with emphasis on inheritance patterns. J Am Dent Assoc 1988 Feb; 96(2):266-275.

9. Ciamponi AL, Frassei VAS. Congenital partial anodontia in the permanent dentition: a study of its prevalence in children living in São Paulo. Rev Pos-Grad 1999 Jul-Sep;6(3):213-217.

10. Koki Ch VO Jr. Congenitally missing teeth: orthodontic management in the adolescent patient. Am J Orthod Dentofac Orthop 2002 Jun;121(6):594-595.

11. Estacia A, Souza MMG. Bilateral congenital absence of maxillary lateral incisor-a clinical case report. J Bras Orthodon Orthop Facial 2000 Jan-Feb;5(25):21-28.

12. Wu H, Feng HL. A survey of number and morphology anomalies in permanent teeth of 6453 youths between 17 and 21 years old. Chinese J Stomatol 2005 Nov;40(6): 489-490.

13. Seabra M, Macho V, Pinto A, Soares D, de Andrade C. A importância das anomalias dentárias de desenvolvimento. Acta Pediatr Port 2008 Jul;39(5):195-200.

14. Ooshima T, Ishida R, Mishima K, Sobue S. The prevalence of developmental anomalies of teeth and their association with tooth size in the primary and permanent dentitions of 1650 Japanese children. Int J Paediatr Dent 1996 Jun;6(2): 87-94.

15. Kook YA, Park S, Sameshima GT. Peg-shaped and small lateral incisors no tathigher risk for root resorption. Am J Orthod Dentofacial Orthop 2003 Mar;123(3):253-258.

16. Boselli G, Pascotto RC. Conoid lateral incisors: diagnosis, plan and direct restorative treatment. R Dent Press Estét 2007 Apr-May-Jun;2(4):89-96.

17. Mondelli J. Estética e cosmética na clínica integrada restauradora. 1st ed. São Paulo, SP: Santos Ed; 2003. p. 546.
18. Dietschi D. Free-hand composite resin restorations: a key to anterior aesthetics. Pract Periodontics Aesthet Dent 1995 Sep; 7(7):15-25.

19. Teixeira MCB, Maia LC, Valença AMG, Mendes VAS. Esthetic correction of a peg-shaped lateral incisor: case report. J Bras Odontopediatr Odontol Bebê 2003 May-Jun;6(31): 230-233.

20. Bandéca MC, Tonetto MR, Kabbach W, Pinto SCS, de Oliveira-OB Jr, Saad JRC. The art of copy-anterior aesthetic restoration. Rev Dental Press Estét 2011 Jan-Mar;8(1):90-103.

21. Lima Filho RMA, et al. Tratamento de Classe II, Divisão 1, com ausência congênita de incisivo lateral superior. Rev Dent Press Ortodon Ortop Facial 2004 Sep-Oct;9(5):95-101.

22. Oliveira AAG, Consolaro A, Henquiques JFC. Relation of partial anodontia and the permanent teeth of Brazilians. I Association of its occurrence with the mesiodistal size of the dental crowns. Rev Odontol Univ São Paulo 1991 Jan-Jun; 5(1):7-14.

23. Macley RJ. An evaluation of smiles before and after orthodontic treatment. Angle Orthod 1993;3(63):183-189.

24. Kina M, Leal FA, Fabre FA, Martin OCL, Coimbra MC, Kina J. Treatment minimally invasive aesthetic restorative through direct technique with composite: case report. Arch Health Invest 2015;4(4):50-55.

25. Clavijo VGR, de Souza NC, Kabbach W, Rigolizzo DS, de Andrade MR. Utilization of self-etching adhesive in direct composite restoration-a clinical protocol. R Dental Press Estét 2006 Oct-Nov-Dec;3(4):34-42.

26. Hirata R, Ampessan RL, Liu J. Reconstruction of anterior teeth with composite resin-sequence of choice and application. J Br Clin Estét Odontol 2001 Jan-Feb;5(25):15-25.

27. De Moura Costa PV, et al. Replacing esthetic restorations: effect of fluorescence composites in aesthetic dentistry. Rev Odontol Bras Central 2014 Jan-Feb;23(67):226-230.

28. Higachi C, Gomes JC, Kina S, de Andrade OS, Hirata R. Planejamento estético em dentes anteriores. In: Miyashita E, Mello AT. Odontologia Estética - Planejamento e técnicas. 2nd ed 2006. 139-154 p.

29. Santezi C, et al. Anterior restorations in bleached teeth: difficulty establishing the color after bleaching. J Contemp Dent Pract 2012 Sep;13(5):735-739.

30. Carrilho EVP, Paula A, Tomaz J. Terapêuticas estéticas integradas no âmbito da dentistaria operatória. Rev Port Estomatol Cir Maxilofac 2007 Jan-Mar;1(48):23-34.

31. Dietschi D. Free-hand bonding in the esthetic treatment of anterior teeth: creating the illusion. J Esthet Dent 1997;9(4): 156-164.

32. Dietschi D. Layering concepts in anterior composite restorations. J Adhes Dent 2001 Spring Mar-Jun;3(1):71-80.

33. World Health Organization (WHO). Mental health: strengthening our response. WHO media centre. [Internet], USA, 2013. [Assessed in 2013 Sep 23]. Disponible in http:// www.who.int/mediacentre/factsheets/fs220/en/. 